UDC 61

\title{
COMPARISON OF THE ACCURACY OF qSOFA AND MEWS SCORE FOR EARLY DETECTION OF SEPSIS
}

\author{
Mahrus $^{1,2^{*}}$, Dradjat Respati ${ }^{2,3}$, Freddy Antonius $^{3}$, Setijowati Nanik ${ }^{4}$ \\ ${ }^{1}$ Emergency Medicine Postgraduate Program, Faculty of Medicine, University of Brawijaya, \\ Indonesia \\ ${ }^{2}$ Saiful Anwar General Hospital, Indonesia \\ ${ }^{3}$ Department of Emergency Medicine, Faculty of Medicine, University of Brawijaya, Indonesia \\ ${ }^{4}$ Department of Public Health, Faculty of Medicine, University of Brawijaya, Indonesia \\ *E-mail: dr mahrus@yahoo.co.id
}

\begin{abstract}
MEWS is a scoring that has been observed for early enforcement of severe sepsis in ED $(78,8 \%$ sensitivity), whereas qSOFA is a scoring that is still debatable in determining suspicious sepsis patients that is recommended by International Consensus Sepsis-3. The objective of this study is to compare the accuracy between newly applied qSOFA score with previously investigated MEWS in predicting early sepsis in septic patients at Emergency Department (ED), Saiful Anwar General Hospital (SAGH), Malang - Indonesia. This is an observational cross-sectional study to assess the accuracy of qSOFA compared to MEWS in determining early diagnosis of sepsis. We collected the data of patients diagnosed with sepsis for 5 months (July to November 2017) at ED in SAGH Malang. There were 74 patients who met our inclusion criteria. We used lactic acid value of $\geq 2$ as cut off value to diagnose patients with sepsis. Our research data shows that to determine sepsis, qSOFA and MEWS (cut off lactate $\geq 2$ ) have sensitivity $93.8 \%$ vs $81.5 \%$, PPV $87.1 \%$ vs $88.3 \%$, Specificity $0 \%$ vs. $22.2 \%$, NPV $0 \%$ vs $14.3 \%$, Accuracy $82.4 \%$ vs. $74.3 \%$. In addition, the comparison of qSOFA and MEWS as diagnostic tests to predict 24-hour mortality in ED has an accuracy of $22 \%$ vs $40.5 \%, 100 \%$ sensitivity vs $94 \%$ and specificity $7.1 \%$ vs. $23 \%$. qSOFA is more accurate than MEWS in determining suspected sepsis patients ( $82.4 \%$ vs $74.3 \%)$ based on lactic acid so that qSOFA can be used as a scoring tool of sepsis at ED Saiful Anwar Hospital. However, qSOFA and MEWS still cannot be used as a scoring tool for predicting 24 -hour mortality in ED due to its very low accuracy (22\% vs $40.5 \%$ ) although MEWS accuracy is higher than qSOFA.
\end{abstract}

\section{KEY WORDS}

Lactate levels, MEWS, qSOFA, sepsis

Sepsis is one of the oldest and most elusive syndromes in the world of medicine (Wiersinga et al., 2014). Sepsis causes millions of deaths worldwide each year (Deutschman and Tracey, 2014). Lactate serum is a marker that describes global tissue hypoperfusion and it's used as the gold standard to assess severity in septic patients. According to existing theory, although patients have not experienced hypotension, tissue perfusion has decreased with the presence of cardiac depression, relative hypovolemia due to vascular endothelial leakage, and decreased vasoregulator mechanism. These result in increased oxygen demands, and lactate is formed as a product of anaerobic metabolism that contributes to mortality (Berger et al., 2013).

In its development, The Third International Consensus Definitions for Sepsis and Septic Shock (Sepsis-3) 2016 define sepsis as life-threatening organ dysfunction caused by dysregulated host response to infection. It recommends qSOFA (quick Sequential related Organ Function Assessment) as the initial screening of patients with sepsis (Seymour et al., and Singer et al 2016). However, qSOFA is not widely used as an initial assessment of patients with sepsis.

In Indonesia, the mortality rate of patients with sepsis is high. In addition, not all hospitals have lactic acid examination which is the gold standard for sepsis predictors. In 
SAGH (SAGH), cases of sepsis ranks 10th out of ten major emergency diseases. Although the prevalence of sepsis decreased from the previous year, it ranked first $(15.91 \%)$ in the top ten causes of death in hospital (Annual Report SAGH, 2016). Modified Early Warning Score (MEWS) is a scoring that has been observed for early diagnosis of severe sepsis in ED (78.8\% accurate, $80.9 \%$ sensitivity). When sepsis can be detected early in ED and treated promptly in accordance with the EGDT (Early Goal Direct Treatment) for sepsis, there is a significant decrease in mortality (Berger et al., 2013).

Defining MEWS and qSOFA Score. The qSOFA criteria were defined as systolic blood pressure $\leq 100 \mathrm{~mm} \mathrm{Hg}$, respiratory rate $\geq 22$ breaths per minute, and altered mental status (defined as either a Glasgow Coma Scale score $\leq 13$ (Seymour et al., and Singer et al 2016). MEWS criteria were defined as systolic blood pressure, Heart rate, temperature, Respiratory rate and AVPU with calculated based on previously published tables (Bulut, M. et., al. 2013)

\section{METHODS OF RESEARCH}

Setting and design. This study was a single-center, cross sectional observational study of patients with clinically diagnosed infection at the ED of Malang Saiful Anwar Hospital from July to November 2017. The hospital is a about 890-bed teaching hospital with approximately 20000 ED visitors per year. This study was approved by the Ethics Committee of our institution. All the patients gave written informed consent.

Patients. Samples were taken by random sampling technique.

Inclusion Criteria:

- Non trauma patients with clinical suspicion of sepsis (fulfilling SIRS criteria for suspected infection);

- Age 15 to 80 years.

Exclusion Criteria:

- Patients with acute heart failure or cardiogenic shock;

- Patients referred from other health facilities that come with attached vasopressor medications or post fluid resuscitation;

- Patients who come with shock conditions due to bleeding..

Data collection. Data on demographic characteristics and vital signs (blood pressure, pulse rate, breathing rate, temperature, GCS/AVPU) were collected. MEWS and qSOFA scores were calculated using the data at enrollment. Lactate serum level of enrolled patients was recorded upon ED arrival.

Data analysis. The data is presented in tabulation with table $2 \times 2$ and assessed for sensitivity, specificity, positive predictive value (PPV), negative predictive value (NPV), and accuracy. The correlation between each independent variable contained in qSOFA and MEWS and lactate acid level were tested by chi square test. If the correlation of each variable obtained was $p<0.25$, the analysis is continued into a logistic regression test with $95 \%$ confidence level, $\alpha=0.05$, significant when $p<0.05$.

Table 1 - Patient Characteristics

\begin{tabular}{|l|l|l|}
\hline Characteristics & $\Sigma$ & $(\%)$ \\
\hline Gender: & 40 & \\
Male & 34 & 54 \\
Female & & 46 \\
\hline Age: & 3 & \\
$15-30$ & 14 & 19 \\
$31-45$ & 18 & 24 \\
$46-60$ & 39 & 53 \\
\hline$>60$ & & \\
\hline
\end{tabular}

\section{RESULTS AND DISCUSSION}

Patient characteristics. There were a total of 105 samples collected from July to November 2017. 32 patients were excluded due to incomplete data. The data from 74 
samples were analyzed males and females are $54 \%$ vs $46 \%$ which the majority of age group of more than 60 years (table 1).

Frequency Distribution. The frequency distribution of each parameter contained in qSOFA varies. The frequency of systolic $>100$ and $\leq 100$ were not too much different (32 vs 42 samples). Respiration rate $\geq 22$ and GCS $<15$ have higher frequency (63 and 59 samples). Of MEWS variables, the most frequent respiratory rate (21-29 times per minute), AVPU (pain response) and temperature $\left(35-38.4{ }^{\circ} \mathrm{C}\right.$ ) were 44,32 and 64 samples respectively. Distribution of patients with lactate values $\geq 2$ were as many as 65 patients, with the remaining 9 have lactate values of $<2$. The distribution frequency of qSOFA $\geq 2$ and MEWS $\geq 5$ is 70 and 60 patients respectively (table 2 ).

Table 2 - The Central Tendency

\begin{tabular}{|l|l|l|l|l|l|}
\hline \multicolumn{1}{|c|}{ Variable } & \multicolumn{1}{c|}{ X } & \multicolumn{1}{c|}{ SD } & \multicolumn{1}{c|}{ Me } & \multicolumn{1}{c|}{ Max } & \multicolumn{1}{c|}{ Min } \\
\hline Age $(\mathrm{n}: 74)$ & 57.97 & 14.587 & 61.00 & 80 & 18 \\
\hline Systole $(\mathrm{n}: 70)$ & 102.86 & 29.286 & 99.00 & 179 & 60 \\
\hline HR $(\mathrm{n}: 73)$ & 112.23 & 30.456 & 112.00 & 218 & 37 \\
\hline RR $(\mathrm{n}: 70)$ & 27.71 & 6.106 & 28.00 & 50 & 16 \\
\hline GCS (n: 74) & 9.85 & 4.255 & 9.50 & 15 & 3 \\
\hline Lactate serum (n: 74) & 5.409 & 4.0628 & 4.050 & 19.0 & 1.1 \\
\hline Temp (n: 74$)$ & 36.742 & 1.0215 & 36.350 & 38.9 & 35.0 \\
\hline
\end{tabular}

$X$ : mean, SD: standard deviation, Me: median, Max: maximum, Min: minimum, HR: heart rate, RR: respiratory rate.

Comparison of qSOFA and MEWS accuracy. The sensitivity of qSOFA in assessing sepsis reflected from $\geq 2$ lactate acid level was $93.8 \%$. Specificity was $0 \%$, PPV $87.1 \%$, and NPV 0\%. PPV approached $90 \%$ and its accuracy was $82.4 \%$. Sensitivity of MEWS in assessing sepsis reflected from lactic acid levels was $81.5 \%$. Specificity was $22.2 \%$, PPV $88.3 \%$, and NPV $14.4 \%$ and its accuracy was $74.3 \%$ (table 3 ).

Correlation between qSOFA and MEWS with Lactic Acid Level. Pearson Chi-Square test on the correlations of qSOFA and MEWS with lactate levels found no significant correlation ( $p>0.05)$. It also did not qualify for regression test.

Table 3 - Comparison of Accuracy between qSOFA and MEWS for early detection of sepsis patients

\begin{tabular}{|l|l|l|l|l|l|}
\hline Criteria & Sensitivity (\%) & Specificity (\%) & PPV & NPV & Accuracy (\%) \\
\hline qSOFA & 93.8 & 0 & 87.1 & 0 & 82.4 \\
MEWS & 81.5 & 22.2 & 88.3 & 14.4 & 74.3 \\
\hline
\end{tabular}

Table 4 - Correlation between qSOFA and MEWS with Lactate level

\begin{tabular}{|c|c|c|c|c|c|}
\hline \multirow{2}{*}{\multicolumn{2}{|c|}{ Criteria }} & \multicolumn{2}{|c|}{ Lactate } & \multirow{2}{*}{$\Sigma$} & \multirow{2}{*}{ P } \\
\hline & & $<2$ & $\geq 2$ & & \\
\hline qSOFA & \begin{tabular}{|l|}
$<2$ \\
$\geq 2$ \\
Total
\end{tabular} & $\begin{array}{l}0 \\
9 \\
9\end{array}$ & $\begin{array}{l}4 \\
61 \\
65 \\
\end{array}$ & \begin{tabular}{|l|}
4 \\
70 \\
74
\end{tabular} & 1.000 \\
\hline MEWS & \begin{tabular}{|l|}
$<5$ \\
$\geq 5$ \\
Total
\end{tabular} & $\begin{array}{l}2 \\
7 \\
9\end{array}$ & $\begin{array}{l}12 \\
53 \\
65\end{array}$ & \begin{tabular}{|l|}
14 \\
60 \\
74
\end{tabular} & .566 \\
\hline
\end{tabular}

Correlations between qSOFA and MEWS with 24-hour Mortality. There is no significant correlation between qSOFA and patient's first 24-hour mortality in the ED of SAGH, so regression test was not conducted (table 5).

Additional. qSOFA and MEWS Score to Predict Patients Mortality. The accuracy of qSOFA and MEWS to predict the patients mortality is $22 \%$ vs $40.5 \%$ (table 5 ). The number of 
patients with qSOFA $\geq 2$ and MEWS $\geq 15$ who died within the first 24 hours were 18 and 17 patients respectively.

Table 5 - Correlation between qSOFA and MEWS with 24-hour Mortality

\begin{tabular}{|c|c|c|c|c|c|}
\hline \multirow{2}{*}{\multicolumn{2}{|c|}{ Criteria }} & \multicolumn{2}{|c|}{ Mortality } & \multirow{2}{*}{ Total } & \multirow{2}{*}{$\mathrm{P}$} \\
\hline & & $<24 \mathrm{~h}$ & Adm & & \\
\hline qSOFA & $\begin{array}{l}<2 \\
\geq 2\end{array}$ & $\begin{array}{l}0 \\
18\end{array}$ & \begin{tabular}{|l|}
4 \\
52
\end{tabular} & $\begin{array}{l}4 \\
70\end{array}$ & .566 \\
\hline Total & & 18 & 56 & 74 & \\
\hline \multirow[t]{2}{*}{ MEWS } & $\geq 5$ & 1 & 13 & 14 & \\
\hline & $<5$ & 17 & 43 & 60 & .096 \\
\hline Total & & 18 & 56 & 74 & \\
\hline
\end{tabular}

Table 6 - Comparison of qSOFA and MEWS score to predict 24-hour mortality

\begin{tabular}{|c|c|c|c|c|c|}
\hline Score & Sensitivity (\%) & Specificity (\%) & PPV & NPV & Accuracy (\%) \\
\hline qSOFA & 100 & 7,1 & 25,7 & 100 & 22 \\
MEWS & 94 & 23 & 28 & 93 & 40.5 \\
\hline
\end{tabular}

\section{DISCUSSION OF RESULTS}

Of the 74 patients sampled, there were more male patients compared to female patients $(54 \%$ vs $46 \%)$. It is associated with steroid sex hormones that affect the inflammatory response of the body. The most frequent age group is between 65 to 84 years, in accordance to a research conducted by Rezende (Rezende et al., 2008).

.From the above comparison results, in terms of accuracy qSOFA has a slightly higher accuracy compared to MEWS, so qSOFA has a better ability compared to MEWS as a screening tool to establish early diagnosis of sepsis (82.3\% accuracy vs $74.3 \%$ ), although the level of confidence is still low (PPV is less than 90\%). MEWS calculations are not much different from previous research results (MEWS in assessing the severity of sepsis reflected by lactic acid levels) by Canaris A 2016 have accuracy of $78.9 \%$.

The total score of qSOFA and MEWS was obtained $p>0,05$ so that there was no significant correlation between either qSOFA or MEWS with lactate serum level, so it can not be continued to regression test. This is consistent with previous research, where increased MEWS score was not always followed by increased lactic acid levels (Adrianus K, 2016). In addition, hyperlactatemia in sepsis may also occur as a result of endotoxemia although systemic perfusion, blood pressure and oxygen delivery are within normal limits (Chertoff, 2015). The correlations between each variable contained in qSOFA and MEWS with lactic acid levels can not be analyzed because the data is not homogeneous.

There was no significant correlation between qSOFA and MEWS with 24 hours mortality in the ED. This is probably because the deaths of septic patients usually occur in 37 days. In a research conducted by Trzeciak et al. in 2006, patients who died in acute phase ( $\leq 3$ days) usually have lactate level of $\geq 4 \mathrm{mmol}$. Another study shows a significant association between increased MEWS scores and 7-day mortality (Kruisselbrinket R et., al 2016). However, we did not evaluate mortality in the ICU and wards.

qSOFA score of $\geq 2$ in $\leq 24$ hours shows higher survival rate compared with hyperlactatemia. This can still indicate a close association or the influence of high lactic acid levels on mortality rates. Besides, the production of lactic acid is also influenced by many factors, including endogenous lactic acid that cannot be measured or predicted and different in each individual.

The qSOFA score has low accuracy than MEWS despite qSOFA has high sensitivity than MEWS in predicting 24-hour mortality. study, One of study National Early Warning Score (NEWS) being the most sensitive score (Churpek, et., Al. 2017). These, may be due to different demographics, number of samples, and different durations of the study. 


\section{REFERENCES}

1. Abraham, E. (2016). New Definitions for Sepsis and Septic Shock: Continuing Evolution but With Much Still to Be Done. JAMA, 315 (8), 757-759.

2. Adrianus, C. (2016). The Effect and Accuracy of Modified Early Warning Score (Mews) Sepsis As Determinant Of Severe Sepsis (Unpublished Thesis). Emergency Medicine Postgraduate Program Faculty of Medicine Brawijaya University, Malang.

3. Angus, D. C., \& Poll, T. V. D. (2013). Severe Sepsis And Septic Shock. The New England Journal Of Medicine, 369 (9), 840-851.

4. Backer, D. D. (2009). Lactic Acidosis. Applied Physiology In Intensive Care Medicine. Berlin: Springer, pp. 89-92.

5. Berger, T., Green, J., Horeczko, T., Hagar, Y., Garg, N., Suarez, A., \& Panacek, E. (2013). Shock Index And Early Recognition Of Sepsis In The Emergency Department: Pilot Study. Western Journal Of Emergency Medicine, 14 (2), 168-174.

6. Blomkalns, A. L. (2007). Lactate - A Marker For Sepsis And Trauma. EmcregInternational, 43-49. Residency Program Director, Department of Emergency Medicine, University of Cincinnati College of Medicine, Cincinnati, OH, Director of CME and Enduring Materials, EMCREG-International, retrieved from www.emcreg.org.

7. Bulut, M., Cebicci, H., Sigirli, D., Sak, A., Durmus, O., Top, A. A., Kaya, S., \& Uz, K. (2013). The Comparison Of Modified Early Warning Score With Rapid Emergency Medicine Score: A Prospective Multicentre Observational Cohort Study On Medical And Surgical Patients Presenting To Emergency Department. Emergency Medicine Journal, 31 (6), 476-481.

8. Chertoff, J., Chisum, M., Simmons, L., King, B., Walker, M., \& Lascano, J. (2015). Prognostic Utility Of Lactate Clearance After Twenty-Four To Forty-Eight Hours Of Early Goal Directed Therapy In The Management Of Sepsis, Severe Sepsis, And Septic Shock. C23. Sepsis: Risk, Recognition, And Resuscitation. Am Thoracic Soc, 191(1), A4006.

9. Churpek, M. M., Snyder, A., Han, X., Sokol, S., Pettit, N., Howell, M. D., \& Edelson, D. P. (2017). Quick Sepsis-Related Organ Failure Assessment, Systemic Inflammatory Response Syndrome, And Early Warning Scores For Detecting Clinical Deterioration In Infected Patients Outside The Intensive Care Unit. American Journal Of Respiratory And Critical Care Medicine, 195(7), 906-911.

10. Dellinger, R. P., Levy, M. M., Rhodes, A., Annane, D. (2013). Surviving Sepsis Campaign: International Guidelines For Management Of Severe Sepsis And Septic Shock 2012. Intensive Care Med, 39(2), 165 - 228.

11. Deutschman, C. S., \& Tracey, K. J. (2014). Sepsis: Current Dogma And New Perspectives. Immunity, 40(4), 463-475.

12. Fuller, B. M., \& Dellinger, R. P. (2012). Laktat Level As A Hemodynamic Marker In The Critically III. Curr Opin Crit Care, 18(3), 0267-272

13. Hall, M. J., Williams, S. N., Defrances, C. J., \& Golosinskiy, A. (2011). Inpatient Care For Septicemia Or Sepsis: A Challenge For Patients And Hospitals. NCHS Data Brief (62), 18.

14. Jansen, T. C., Bommel, J., Mulder, P. G., Rommes, J. H., Schieveld, S. J. M. \& Bakker, J. (2008). The Prognostic Value Of Blood Lactate Levels Relative To That Of Vital Signs In The Pre-Hospital Setting: A Pilot Study. Critical Care, 12(6), 1-7

15. Jun-Yu Wang, M., Yun-Xia, C., Shu-Bin, G., Xue, M., \& Peng, Y. (2016). Predictive Performance Of Quick Sepsis-Related Organ Failure Assessment For Mortality And Icu Admission In Patients With Infection At The Ed. The American Journal Of Emergency Medicine, 34(9), 1788-1793.

16. Kruisselbrink, R., Kwizera, A., Crowther, M., Fox-Robichaud, A., O'shea, T., Nakibuuka, J., Ssinabulya, I., Nalyazi, J., Bonner, A., \& Devji, T. (2016). Modified Early Warning Score (Mews) Identifies Critical Illness Among Ward Patients In A Resource Restricted Setting In Kampala, Uganda: A Prospective Observational Study. Plos One, 11(3), E0151408. 
17. Leach, R. M., \& Treacher, D. F. (2002). The Pulmonary Physician In Critical Care 2: Oxygen Delivery And Consumption In The Critically III. Thorax, 57(2), 170 - 177.

18. Lyle, N. H., Pena, O. M., Boyd, J. H., \& Hancock, R. E. W. (2014). Barriers To The Effective Treatment Of Sepsis: Antimicrobial Agents, Sepsis Definitions, And HostDirected Therapies. Annals Of The New York Academy Of Sciences, 1323(1), 101-114.

19. Malmir, J., Bolvardi, E., \& Aghaee, M. A. (2014). Serum Lactate Is A Useful Predictor Of Death In Severe Sepsis And Septic Shock. Reviews In Clinical Medicine, 1(3), 97-104.

20. Martin, G. S., Mannino, D. M., Eaton, S. \& Moss, M. 2003. The Epidemiology Of Sepsis In The United States From 1979 Through 2000. The New England Journal Of Medicine, 348(16), 1546-1554.

21. Mattu, A. (2017). Severe Sepsis Care In The Emergency Department. Emergency Medicine Clinics Of North America, 35(1), 15

22. Mayr, F. B., Yende, S., \& Angus, D. C. (2014). Epidemiology Of Severe Sepsis. Virulence, 5(1), 4-11.

23. Nguyen, H. B., Rivers, E. P., Abrahamian, F. M., Moran, G. J., Abraham, E., Trzeciak, S., Huang, D. T., Osborn, T., Stevens, D., \& Talan, D. A. (2006). Severe Sepsis And Septic Shock: Review Of The Literature And Emergency Department Management Guidelines. Annals Of Emergency Medicine, 48(1), 28-54.

24. Phypers, B., \& Pierce, J. T. (2006). Lactate Physiology In Health And Disease. British Journal Of Anaesthesia 6(3), 128-132.

25. Pradipta, I. S., Sandiana, A. T., Halimah, E., Diantini, A., Lestari, K., \& Abdulah, R. (2013). Microbial And Resistance Profile In Isolate From Adult Sepsis Patients: An Observational Study At An Indonesian Private Hospital During 2009-2012. International Journal Of Pharmaceutical Sciences Review And Research, 19(2), 24-29.

26. Quinton, S. (2011). Adult Modified Early Warning Score (Mews) Policy \& Escalation Pathway V3.0. In: Trust, H. O. E. N. F. (Ed.). United Kingdom: Heart Of England NHS Foundation Trust.

27. Sandesc, D. (2003). Sepsis: A Review (I). Retrieved from Http://Www.Tmj.Ro/Article.Php?

28. Sastroasmoro, S., \& Ismael, S. (1995). Dasar - Dasar Metodologi Penelitian. Jakarta: Binarupa Aksara.

29. Schaechter, M., Engleberg, N. C., Dirita, V., \& Dermody, T. S. (2013). Mechanisms Of Microbial Disease. United State Of America: Lippincott Williams \& Wilkins.

30. Seymour, C., Liu, V., Iwashyna, T., Brunkhorst, F., Rea, T., Scherag, A., Rubenfeld, G., Kahn, J., Shankar-Hari, M., \& Singer, M. (2016). Assessment Of Clinical Criteria For Sepsis: For The Third International Consensus Definitions For Sepsis And Septic Shock (Sepsis-3). Jama, 315 (8),762.

31. Singer, M., Deutschman, C. S., Seymour, C. W., Shankar-Hari, M., Annane, D., Bauer, M., Bellomo, R., Bernard, G. R., Chiche, J.-D., \& Coopersmith, C. M. (2016). The Third International Consensus Definitions For Sepsis And Septic Shock (Sepsis-3). Jama, 315(8), 801-810.

32. Soong, J., \& Soni, N. (2012). Sepsis: Recognition and Treatment. CME Acute Medicine, 12(3), 276-280.

33. Soreng, K., \& Levy, H. R. (2011). Procalcitonin: An Emerging Biomarker Of Bacterial Sepsis. Clinical Microbiology Newsletter, 33(22), 171-178.

34. Subbe, C., Kruger, M., Rutherford, P., \& Gemmel, L. 2001. Validation Of A Modified Early Warning Score In Medical Admissions. Qjm, 94(10), 521-526.

35. Sutton, J. P., \& Friedman, B. (2013). Trends In Septicemia Hospitalizations And Readmissions In Selected Hcup States, 2005 And 2010. Healthcare Cost and Utilization Project (Hcup) Statistical Briefs. United State of America: National Center For Biotechnology Information.

36. Torio, C. M., \& Andrews, R. M. (2013). National Inpatient Hospital Costs: The Most Expensive Conditions By Payer 2011. United State Of America: National Center For Biotechnology Information. 
37. Trzeciak, S., Dellinger, R. P., Chansky, M. E., Arnold, R. C., Schorr, C., Milcarek, B., Hollenberg, S. M., \& Parrillo, J. E. (2007). Serum Lactate As A Predictor Of Mortality In Patients With Infection. Intensive Care Medicine, 33(6), 970-977.

38. Washington, J. (2014). Initiating Mews In The ER - Early Sepsis Recogntion. Retrieved from www.nhffa.org.

39. Wiersinga, W. J., Leopold, S. J., Cranendonk, D. R., \& Poll, T. V. D. (2014). Host Innate Immune Responses To Sepsis. Virulence, 5(1), 36-44. 